\title{
Single-Molecule Electrochemical Gating in lonic Liquids
}

Kay, Nicola J.; Higgins, Simon J.; Jeppesen, Jan O.; Leary, Edmund; Lycoops, Jess; Ulstrup, Jens; Nichols, Richard J.

Published in:

Journal of the American Chemical Society

Link to article, DOI:

10.1021/ja307407e

Publication date:

2012

Document Version

Publisher's PDF, also known as Version of record

Link back to DTU Orbit

Citation (APA):

Kay, N. J., Higgins, S. J., Jeppesen, J. O., Leary, E., Lycoops, J., Ulstrup, J., \& Nichols, R. J. (2012). SingleMolecule Electrochemical Gating in lonic Liquids. Journal of the American Chemical Society, 134(40), 1681716826. https://doi.org/10.1021/ja307407e

\section{General rights}

Copyright and moral rights for the publications made accessible in the public portal are retained by the authors and/or other copyright owners and it is a condition of accessing publications that users recognise and abide by the legal requirements associated with these rights.

- Users may download and print one copy of any publication from the public portal for the purpose of private study or research.

- You may not further distribute the material or use it for any profit-making activity or commercial gain

- You may freely distribute the URL identifying the publication in the public portal 


\title{
Single-Molecule Electrochemical Gating in Ionic Liquids
}

\author{
Nicola J. Kay, ${ }^{\dagger}$ Simon J. Higgins, ${ }^{* \dagger}{ }^{\dagger}$ Jan O. Jeppesen, ${ }^{\ddagger}$ Edmund Leary, ${ }^{\dagger, \perp}$ Jess Lycoops, ${ }^{\ddagger}$ Jens Ulstrup, ${ }^{\S}$
} and Richard J. Nichols*,†

${ }^{\dagger}$ Department of Chemistry, Donnan and Robert Robinson Laboratories, University of Liverpool, Liverpool L69 7ZD, U.K.

${ }^{\ddagger}$ Department of Physics, Chemistry, and Pharmacy, University of Southern Denmark, Campusvej 55, DK-5230 Odense M, Denmark

${ }^{\S}$ Department of Chemistry and NanoDTU, Technical University of Denmark, DK2800 Kgs. Lyngby, Denmark

\section{Supporting Information}

ABSTRACT: The single-molecular conductance of a redox active molecular bridge has been studied in an electrochemical singlemolecule transistor configuration in a room-temperature ionic liquid (RTIL). The redox active pyrrolo-tetrathiafulvalene (pTTF) moiety was attached to gold contacts at both ends through $-\left(\mathrm{CH}_{2}\right)_{6} \mathrm{~S}-$ groups, and gating of the redox state was achieved with the electrochemical potential. The water-free, room-temperature, ionic liquid environment enabled both the monocationic and the previously inaccessible dicationic redox states of the pTTF

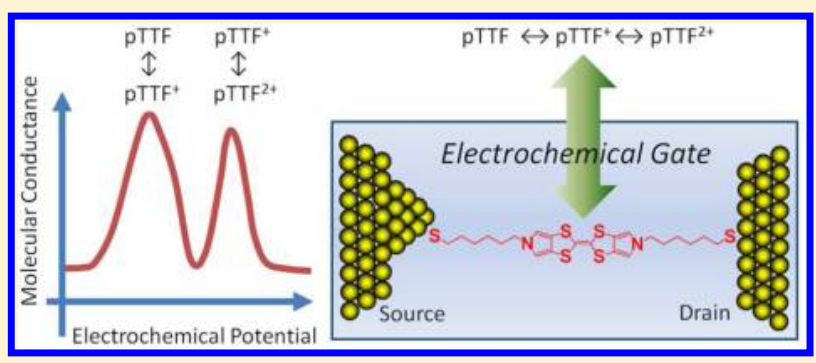
moiety to be studied in the in situ scanning tunneling microscopy

(STM) molecular break junction configuration. As the electrode potential is swept to positive potentials through both redox transitions, an ideal switching behavior is observed in which the conductance increases and then decreases as the first redox wave is passed, and then increases and decreases again as the second redox process is passed. This is described as an "off-on-off-onoff" conductance switching behavior. This molecular conductance vs electrochemical potential relation could be modeled well as a sequential two-step charge transfer process with full or partial vibrational relaxation. Using this view, reorganization energies of $\sim 1.2 \mathrm{eV}$ have been estimated for both the first and second redox transitions for the pTTF bridge in the 1-butyl-3methylimidazolium trifluoromethanesulfonate (BMIOTf) ionic liquid environment. By contrast, in aqueous environments, a much smaller reorganization energy of $\sim 0.4 \mathrm{eV}$ has been obtained for the same molecular bridge. These differences are attributed to the large, outer-sphere reorganization energy for charge transfer across the molecular junction in the RTIL.

\section{INTRODUCTION}

The single-molecule transistor is an attractive concept in molecular electronics in which a single molecule is attached between two terminals, while the energy levels in the resulting molecular bridge are controlled by a third proximal electrode. In this three-electrode configuration, molecular energy levels in the molecular bridge are tuned by the gate bias of this third electrode. The gate voltage $\left(V_{\mathrm{g}}\right)$ can be used to tune wave functions and particularly redox energy states of the molecular bridge with respect to the contacts, with consequent control over the junction conductance. Positioning of three electrodes in intimate proximity of a molecule is challenging. This has been achieved for solid-state configurations using planar devices with a metal gate electrode underlying and separated from the source-drain contact pair by a thin dielectric film, ${ }^{1-5}$ as schematically illustrated in Figure 1a. Such three-terminal devices for single-molecule studies have been successfully studied, although it is not possible to precisely control the degree of molecule-gate electrode coupling, and there are considerable technical challenges in achieving the precise source-drain gap spacing which matches molecular lengths necessary for good electrical coupling of the molecule to both leads. There is also the additional challenge of avoiding the evaporation of metal grains in the electrode gap during metal evaporation in the junction fabrication, which is detrimental to defined electrical characterization of molecular junctions. Nevertheless, through careful device preparation, a powerful platform for the characterization of quantum transport through molecules is achieved. ${ }^{1-5}$ Strong gate coupling to the molecule has been observed and transport as a function of gate and source-drain voltages analyzed. It has been demonstrated that a gate electrode can be introduced into mechanically controlled break junctions (Figure 1b). ${ }^{6}$ Although these may have lower gate coupling than the planar configurations, they permit more precise control over the source-drain gap through bending of the underlying substrate.

An alternative approach to the solid-state gating illustrated in Figure $1 \mathrm{a}, \mathrm{b}$ is to use an electrochemical gate. In this setup the bridge energetics are controlled through the electrochemical potential for the molecular device immersed in electrolyte solution relative to a reference electrode. This is typically achieved in a four-electrode electrochemical setup with counter- and reference electrodes serving alongside the source and drain electrodes (both of which are the working electrodes of the electrochemical cell). A schematic illustration of

Received: July 27, 2012

Published: September 17, 2012 


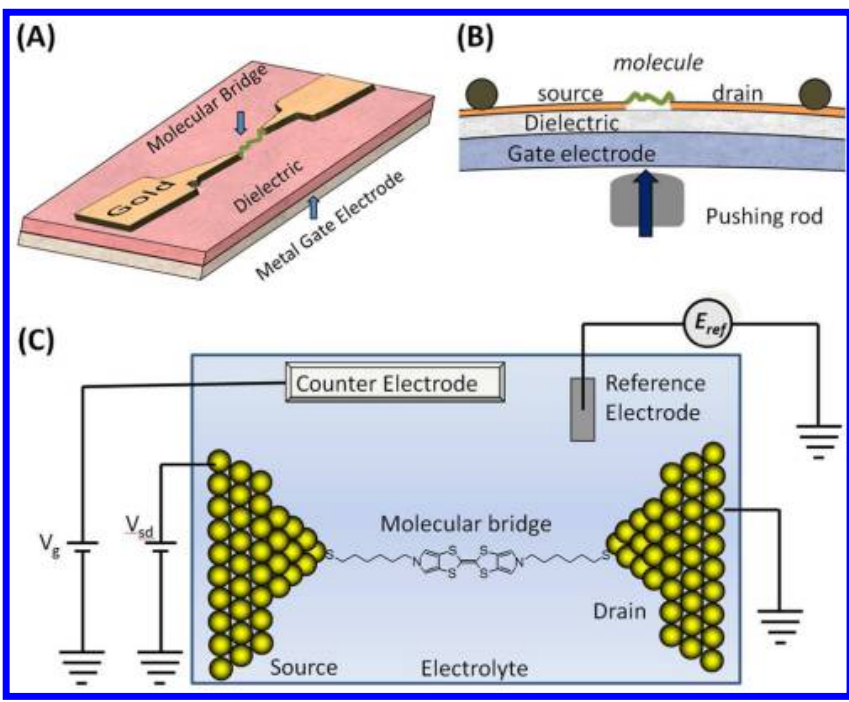

Figure 1. (a) Planar molecular junctions with solid-state gating, (b) mechanically formed break junctions with solid-state gating, and (c) an electrochemical molecular break junction with electrolyte gating. In the electrochemical setup the counter- and reference electrodes which are employed for electrochemical gating are remote from the molecular junction; however, since in strong electrolytes the Debye screening length can be extremely short (down to a few ångstroms) the screening of charge on the molecular bridge is much shorter than can be generally achieved in the solid-state setup.

electrolyte gating of a single molecule is shown in Figure 1c. In strong electrolytes the Debye screening length can be extremely short, i.e., a few ångstroms, so that, although the counter- and reference electrodes are physically far from the molecular bridge, any charge on the molecular bridge is screened by charge in solution at a distance much shorter than can be generally achieved in the solid-state setup of Figure $1 \mathrm{a}$ and $\mathrm{b}$. For instance, if a $0.5 \mathrm{~V}$ potential difference exists across an electrochemical double layer thickness of $0.5 \mathrm{~nm}$, then this results in a mean electric field strength across this double layer region of $10^{9} \mathrm{~V} \mathrm{~m}^{-1}$. The result is a strong and reproducible gate field which is sufficient to significantly modulate the current across single molecules. ${ }^{7}$ There are now a number of reports of electrolyte-gated single-molecule transistors. In a seminal publication in 1996 Tao used scanning tunneling microscopy (STM) to study tunneling through $\mathrm{Fe}$ (III)protoporphyrin (FePP) adsorbed flat on a highly ordered pyrolytic graphite (HOPG) electrode. ${ }^{8}$ At the electrode potential where FePP was reduced, an enhanced tunneling current was observed to flow between the tip and substrate through adsorbed FePP. This "resonance condition" was interpreted as arising from alignment of the Fermi level of the metal electrode with the lowest unoccupied molecular level (LUMO) of FePP. With the advent of STM molecular junction techniques (e.g., the in situ break junction $(\mathrm{BJ})^{9}$ or $I(s)^{10}$ techniques) it later became possible to study electrolyte-gated molecular conductance where the molecular bridge is connected at both ends to metal contacts (at one end to the substrate surface and at the other to an STM tip). ${ }^{10}$ Viologen (bipyridinum) molecular bridges were the first electrochemically gated systems to be studied using this method by Haiss et al., ${ }^{10}$ and this has also been the theme of a number of subsequent studies. ${ }^{11-15}$ A number of other electrochemically gated systems have been investigated, mostly with electrochemical in situ STM approaches, both with the molecule attached at either end to both tip and substrate, or attached at one end only, usually to the substrate. The latter approach can be compared to classic scanning tunneling spectroscopy (STS) with an STM tip positioned above an adsorbed molecular adlayer; however, in the electrolyte environment the electrochemical potential can be swept as well as the tip-to-substrate bias voltage. Other single-molecule systems studied with electrochemical gating and the in situ $\mathrm{BJ}$ or $I(s)$ techniques have included oligophenylene-ethynylenes, ${ }^{16}$ various perylene tetracarboxylic diimide derivatives, ${ }^{17,18}$ oligo-anilines, ${ }^{19-21}$ and a pyrrolo-tetrathiafulvalene (pTTF) derivative, ${ }^{22}$ while systems studied by electrochemical STS include transition metal complexes, $^{23-27}$ viologens, ${ }^{13,15}$ and redox metalloproteins. ${ }^{28,29}$ Electrochemical gating has also been achieved in mechanically controlled break junctions. ${ }^{30}$

Both the in situ electrochemically gated molecular break junction and electrochemical STS configuration can be modeled in terms of the redox active moiety positioned within the nanogap between the metal electrode substrate and metal STM tip. At a qualitative level, the response of this junction while the electrochemical potential is swept can be understood by considering the relative positions of the Fermi levels of the enclosing metal contacts and the oxidized and reduced states of the redox moiety of the molecule. As shown in Figure 2, as

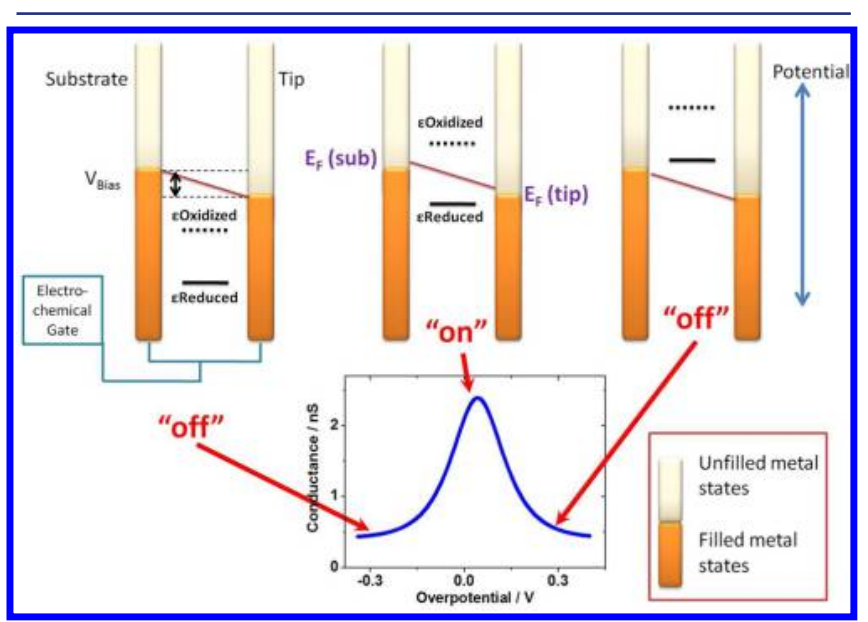

Figure 2. Model for two-step charge transfer across a molecular bridge in an STM nanogap. The energy level models at the top of the figure show alignment for negative (left), zero (center) and positive (right) overpotentials. The lower plot illustrates tunneling current vs overpotential, with a peak near to zero overpotential.

these are swept from an out of resonance condition (left panel) and into the "Fermi window" defined by the two metal electrodes (center panel), the conductance increases and then decreases again as the energy levels move out of resonance (right panel). Independently of mechanistic details, a maximum is therefore expected in the conductance vs electrochemical potential relation sketched in Figure 2. Whether this maximum occurs near zero overpotential $(\eta)$ for the redox transition or whether it is displaced by the reorganization energy depends on the mechanism for charge transfer across the bridge. In the sequential two-step electron/hole transfer model including vibrational relaxation introduced by Kuznetsov and Ulstrup (the KU model), ${ }^{31-36}$ the maximum is expected close to $\eta=0$ (although this depends on the potential distribution in the tunneling gap, which is in turn controlled by the ionic strength), whereas in a resonance tunneling model of 
Schmickler et al. ${ }^{37,38}$ for electron transfer through a redox center, ${ }^{38}$ the maximum is displaced along the overpotential axis by the reorganization free energy. In the electrochemical scanning tunneling spectroscopy configuration (i.e., STM tip not chemically attached to the molecule) there is now a collection of examples displaying a clear maximum in their tunneling current $\left(I_{\text {tunneling }}\right)$ vs electrochemical potential relations. ${ }^{13,15,23-27,29,39}$ These have been well-modeled in terms of the KU relationship for two-step electron/hole transfer through the redox center in the STM-substrate gap. The situation is far from clear for measurements made in the in situ electrochemically gated BJ configuration (i.e., when the electrochemically active bridge molecule is chemically attached at one end to the STM tip and at the other end to the substrate). Typically a far more complex $I_{\text {tunneling }}$ vs electrochemical potential response is observed. ${ }^{10,12,15}$ For instance, for the viologen 6V6 (Figure 3) the molecular conductance

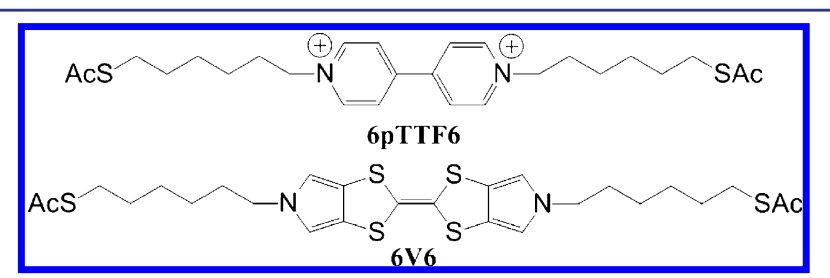

Figure 3. Structures of the redox-active molecular wires 6pTTF6 and 6 V6. The " 6 " in each case refers to the $\left(\mathrm{CH}_{2}\right)_{6} \mathrm{SAc}$ groups, where $\mathrm{SAc}$ is the thioacetate moiety (i.e., a protected thiol group).

increases upon reducing the viologen group, but it remains at this higher conductance value even as the electrode potential is swept more negative, leading to a sigmoidal ${ }^{10,12,15}$ rather than the bell-shaped profile depicted in Figure 2. Other systems in the molecular break junction configuration also exhibit $I_{\text {tunneling }}$ vs electrochemical potential responses which do not easily accord with the KU or Schmickler models for electron transfer across the redox active bridge unless additional features such as gated charge transfer are introduced, as discussed by Haiss et al. ${ }^{12}$ An exception is the pTTF system in aqueous electrolyte reported by Leary et al., ${ }^{22}$ which, at least qualitatively, appears to give a response which accords with either the $\mathrm{KU}$ or Schmickler models. However, detailed analysis of this system has not been made with respect to either model.

In this report, we present a detailed new investigation of the pTTF redox group, incorporated in the molecule 6pTTF6 (Figure 3). Single-molecule bridges are formed using an STM method (the $I(s)$ method $^{10}$ ) in which contact between the STM tip and gold substrate surface is avoided. This is achieved in an electrochemical environment so that the $I_{\text {tunneling }}$ vs electrochemical potential relation can be constructed. Although STS has been successfully conducted in ionic liquids with electrochemical potential control, ${ }^{23}$ these present measurements are the first of electrochemical single-molecule conductance measurements made using the $I(s)$ or BJ methods in a anhydrous ionic liquid environment. This enables the study of the molecular conductance over a much wider electrochemical potential range, as it is first switched to its monocationic state $\left(\mathrm{pTTF}^{+\bullet}\right)$ and then to its dicationic state $\left(\mathrm{pTTF}^{2+}\right)$. The $I_{\text {tunneling }}$ vs electrochemical potential responses are analyzed in terms of the KU model for two-step charge transfer through the redox center, for both the first (pTTF $\rightarrow$ $\mathrm{pTTF}^{+\bullet}$ ) and second redox transition $\left(\mathrm{pTTF}^{+} \rightarrow \mathrm{pTTF}^{2+}\right)$. From the KU model an estimation of the reorganization free energy can be obtained. Since the first redox transition (but not the second) can also be observed in aqueous electrolytes, a value for the reorganization free energy for $\mathrm{pTTF} \rightarrow \mathrm{pTTF}^{+\bullet}$ can also be obtained under aqueous conditions. The values for reorganization free energy for the two different solvent environments are found to differ greatly. Reasons for this difference are discussed.

\section{EXPERIMENTAL METHODS}

Synthesis. The compound 6pTTF6 was synthesized by a published route. $^{22,41}$

Electrochemistry Experiments. All cyclic voltammetry measurements were performed on Ecochemie Autolab potentiostats; either the PGSTAT 20 or PGSTAT 30 model with the corresponding Autolab GPES software. A three-electrode setup was used, with a $\mathrm{Au}(111)$ working electrode (WE), Pt wire mesh counter-electrode (CE) and a quasi-reference electrode consisting of a Pt wire. The electrode surface area of the $\mathrm{Au}(111) \mathrm{WE}$ used was the measured geometric area in contact with the electrolyte. The glass electrochemical cell was cleaned in a 1:1 mixture of $\mathrm{H}_{2} \mathrm{SO}_{4}$ and $\mathrm{HNO}_{3}$ prior to use and rinsed several times in Milli- $\mathrm{Q}$ water. The $\mathrm{CE}$ and WE were flame annealed prior to use. The cell was dried in an oven before use.

The RTIL 1-butyl-3-methylimidazolium trifluoromethanesulfonate (BMIOTf) was purchased from Iolitec. This was dried by heating to 110-120 ${ }^{\circ} \mathrm{C}$ with anhydrous $\mathrm{N}_{2}$ flow for a minimum of $1 \mathrm{~h}$ immediately prior to use and was treated with $3 \AA$ molecular sieves. Gnahm and Kolb have previously reported on the purification of ionic liquids using a molecular sieve with $3 \AA$ pore size. ${ }^{82}$ For cyclic voltammetry the airtight $\mathrm{EC}$ cell was assembled in a glovebox containing an inert $\mathrm{N}_{2}$ atmosphere. A range of scan rates is investigated. To enable conversion to the $\mathrm{Fc} / \mathrm{Fc}^{+}$potential scale, ferrocene was added to the BMIOTf solutions at the conclusion of the experiments. Metallocene derivatives, such as ferrocene (when soluble), are commonly used as effective voltammetric reference standards for ionic liquids. ${ }^{50,83}$

For electrochemistry of 6pTTF6 monolayers, the Au(111) working electrode was immersed in a $1 \mathrm{mM}$ 6pTTF6 in BMIOTf solution for approximately $24 \mathrm{~h}$, then rinsed with BMIOTf before being inserted into the electrochemical cell containing fresh BMIOTf electrolyte. In a manner similar to that of the solution-based voltammetry, $1 \mathrm{mM}$ ferrocene was added to the BMIOTf electrolyte at the conclusion of the experiments to calibrate the potential scale to the $\mathrm{Fc} / \mathrm{Fc}^{+}$redox couple.

Single-Molecule Conductance Measurements. All of the single-molecule experiments were performed using an Agilent 2500 or 5500 STM controller in conjunction with the Agilent Picoscan 5.3.3 software. Due to the sensitivity of the RTIL to air/water, an environmental chamber was fitted to the STM head, to maintain an anhydrous, inert $\mathrm{N}_{2}$ atmosphere. The environmental chamber also contained a small amount of silica desiccant. Au STM tips were prepared using $0.25 \mathrm{~mm} \mathrm{Au}$ wire (99.99\%, Goodfellows) which were electrochemically etched in a $1: 1$ solution of $\mathrm{HCl}$ and ethanol at approximately $+7.0 \mathrm{~V}$. The Au tips were then coated with a layer of Apiezon wax, ensuring that only the very end of the tip was exposed. Commercial gold-on-glass substrates (Arrandee) were flame annealed for approximately $5 \mathrm{~min}$ prior to use. BMIOTf was dried at $120{ }^{\circ} \mathrm{C}$ under vacuum for approximately $18 \mathrm{~h}$ prior to use and treated with $3 \AA$ molecular sieves. For the STM measurements, 6pTTF6 monolayers were formed on the gold-on-glass substrate by immersing the substrate in a $1 \mathrm{mM}$ solution of 6pTTF6 in BMIOTf for approximately $5 \mathrm{~min}$. This was then rinsed with BMIOTf and then ethanol and blown dry using $\mathrm{N}_{2}$. In order to perform STM under electrochemical potential control, a Teflon cell was used in the STM setup and a Pt quasireference and counter-electrode system employed. Once BMIOTf had been added to the STM cell, the environmental chamber was purged with anhydrous $\mathrm{N}_{2}$ for approximately $16 \mathrm{~h}$ prior to measurements.

The STM $I(s)$ technique was employed for single-molecule conductance determination with a set-point current $\left(I_{0}\right)$ of $20 \mathrm{nA}$ and a sample bias voltage $\left(V_{\text {BIAS }}\right)$ of $+0.6 \mathrm{~V}$. To record $I(s)$ curves, the 
tip was withdrawn from the set-point distance by $4 \mathrm{~nm}$ with a retraction duration of $0.1 \mathrm{~s}$. Those scans which showed molecular junction formation were plotted into a histogram, which was used to find the molecular conductance. The sample potential was varied between $-0.6 \mathrm{~V}$ and $+0.5 \mathrm{~V}$, with respect to the Pt quasi-reference electrode. The initial tip-to-sample distance $s_{0}$ was approximated using the method described previously by Haiss et al. ${ }^{61}$

\section{RESULTS}

Cyclic Voltammetry in Ionic Liquid. The redox active molecular bridge 6pTTF6 (Figure 3) exhibits two reversible redox reactions, one of which is in the potential range available also in aqueous electrolytes. ${ }^{22,40-42}$ The two electrochemical redox reactions interconvert the neutral $6 \mathrm{pTTF} 6^{\circ}$, the radical cation $6 \mathrm{pTTF}^{+\bullet}$ and the dication $6 \mathrm{pTTF}^{2+}$. Its stability in three redox states makes $6 \mathrm{pTTF} 6$ an attractive system to study from the viewpoint of electrochemical gating. The $\left(\mathrm{CH}_{2}\right)_{6} \mathrm{SAc}$ chains at either end of 6pTTF6 allow the molecule to be tethered between two electrodes, without the complications from isomer mixtures that inevitably arise with other similarly functionalized TTF molecules in single-molecule junction studies. $^{41,42}$ The $n$-hexyl linkers act as tunneling barriers to the pTTF moiety in the center of the molecule, effectively electronically decoupling it from the gold electrodes. The thioacetate termini are protecting groups for the thiol end groups, which are lost upon surface adsorption to form the $\mathrm{Au}-\mathrm{S}$ contact. ${ }^{43}$

Electron-rich TTF derivatives can undergo protonationinduced oxidative degradation in the presence of water..$^{44,45}$ Moreover, only the first redox reaction ( $\mathrm{pTTF} \rightarrow \mathrm{pTTF}^{+\bullet}$ ) is accessible in aqueous electrolyte in the potential 'window' within which adsorbed thiols on gold are stable. We therefore sought a water-free electrochemical environment in which to study the single-molecule conductance of 6pTTF6 in the absence of water. Volatile nonaqueous solvent-based electrolytes are incompatible with our STM instrumentation. Ionic liquids offer a number of positive attributes for electrochemical studies, notably their wide potential window and high conductivity; additionally, their extremely low volatility makes them appropriate for use with the STM..$^{23,46-51}$ They have been shown to be suitable environments for in situ STM imaging, ${ }^{52-57}$ scanning tunneling spectroscopy ${ }^{23,52,55}$ and STM-based single-molecule conductance measurements. ${ }^{58}$ The room-temperature ionic liquid (RTIL) 1-butyl-3-methylimidazolium trifluoromethanesulfonate (BMIOTf) was used in this study. Solution voltammetry of a $1 \mathrm{mM}$ solution of 6pTTF6 dissolved in anhydrous BMIOTf recorded over sweep rates ranging from 20 to $1000 \mathrm{mV} \mathrm{s}^{-1}$ is shown in Figure 4. For these solution voltammetry experiments the thioacetate protected 6pTTF6 was dissolved in the anhydrous BMIOTf, and no $24 \mathrm{~h}$ surface adsorption step was employed, unlike as described in the next paragraph for monolayers of 6pTTF6 on $\mathrm{Au}(111)$. Two reversible redox waves are seen, as observed for related TTF derivatives in nonaqueous electrolytes by Jeppesen and Becher. ${ }^{41}$ The peak separations $\left(\Delta E_{\mathrm{p}}\right)$ are $(62 \pm 9) \mathrm{mV}$ for the first redox wave and $(61 \pm 5) \mathrm{mV}$ for the second redox wave, consistent with one electron processes.

Cyclic voltammetry has also been performed for monolayers of 6pTTF6 on $\mathrm{Au}(111)$ in BMIOTf. Monolayer formation was achieved over $24 \mathrm{~h}$ immersion as described in the Experimental Methods section. As for the solution voltammetry, two clear redox transitions are also seen for a 6pTTF6 monolayer on an $\mathrm{Au}(111)$ substrate in a BMIOTf electrolyte (Figure 5). The

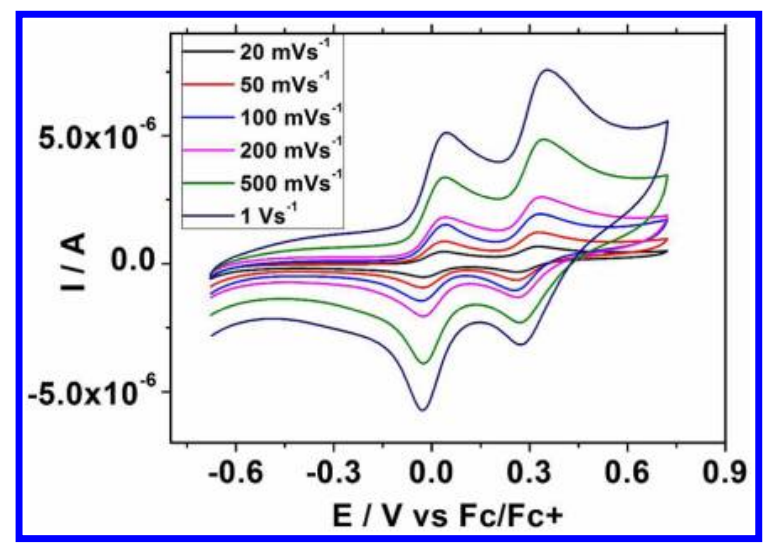

Figure 4. Cyclic voltammograms of a $1 \mathrm{mM}$ solution of 6pTTF6 recorded in BMIOTf (at room temperature), protected under nitrogen. Solution voltammetry recorded at scan rates between 20 $\mathrm{mV} \mathrm{s}^{-1}$ and $1 \mathrm{~V} \mathrm{~s}^{-1}$, as marked. Both redox waves fit the expected peak current $\left(i_{\text {peak }}\right)$ vs square root of the scan rate $\left(\nu^{0.5}\right)$ behavior for a solution-based process $\left(i_{\text {peak }}\right.$ vs $\nu^{0.5}$ gives $r=0.996$ and $r=0.994$ for the first and second oxidation waves, respectively).

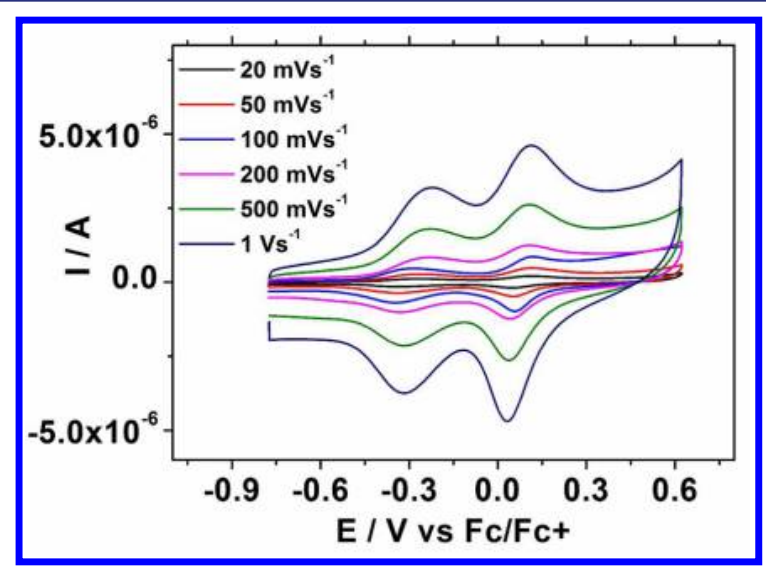

Figure 5. Cyclic voltammograms of a 6pTTF6 monolayer on $\mathrm{Au}(111)$ recorded in BMIOTf at (at room temperature), protected under nitrogen. Surface voltammetry recorded at scan rates between $20 \mathrm{mV}$ $\mathrm{s}^{-1}$ and $1 \mathrm{~V} \mathrm{~s}^{-1}$, as indicated. Both redox waves fit the expected peak current $\left(i_{\text {peak }}\right)$ vs the scan rate $(\nu)$ behavior for a surface based process $\left(i_{\text {peak }}\right.$ vs $\nu$ gives $r=0.997$ and $r=0.996$ for the first and second oxidation waves, respectively).

charges associated with these two peaks are 64 and $59 \mu \mathrm{C} \mathrm{cm}^{-2}$, giving a molecular coverage of $\left(6.6 \times 10^{-10}\right)$ and $\left(6.1 \times 10^{-10}\right)$ $\mathrm{mol} \mathrm{cm}^{-2}$, respectively). These correspond to molecular footprints of 0.25 and $0.27 \mathrm{~nm}^{2}$, respectively. This is consistent with X-ray crystal structure data reported for a related bis(alkanoyl) pTTF compound. ${ }^{59}$ Monolayer formation by 6pTTF6 was also characterized spectroscopically by polarization modulation infrared reflection absorption spectroscopy (see Supporting Information).

In Situ STM. The $I(s)$ technique ${ }^{10}$ was used to determine the single-molecule conductance of 6pTTF6 at various electrochemical potentials, in BMIOTf. The $I(s)$ measurements were performed in an environmental chamber purged with anhydrous $\mathrm{N}_{2}$ gas and $I(s)$ scans were recorded by setting the current set-point and bias voltage, and then rapidly retracting the $\mathrm{Au}$ tip at a rate of $41 \mathrm{~nm} \mathrm{~s}^{-1}$. For a given electrochemical potential, approximately $5 \%$ of the $I(s)$ scans contained a current plateau indicative of molecule(s) bridging the gap between the tip and substrate. At least 500 such scans were 
collected at each electrochemical potential. Representative $I(s)$ scan showing current steps as the molecular bridge is broken are illustrated in Figure 6.

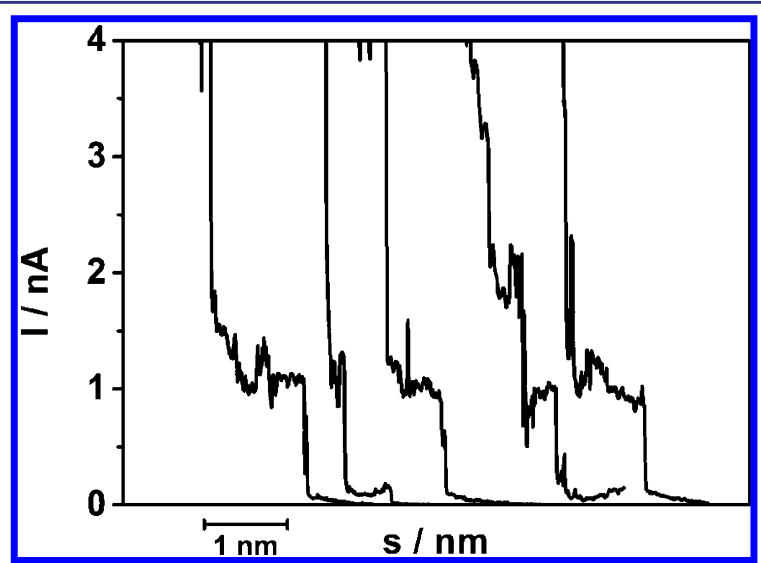

Figure 6. Examples of $I(s)$ scans of 6pTTF6 obtained at room temperature in BMIOTf at a sample potential of $+0.04 \mathrm{~V}\left(\mathrm{vs} \mathrm{Fc} / \mathrm{Fc}^{+}\right)$.

Every $I(s)$ scan which contained a plateau was used in the histogram analysis. Six examples of histograms recorded for electrochemical potentials of $-0.43,-0.33,-0.08,0.04,0.12$, and $0.32 \mathrm{~V}$ vs the $\mathrm{Fc} / \mathrm{Fc}^{+}$reference scale are shown in Figure 7 $\left(\mathrm{Fc} / \mathrm{Fc}^{+}\right.$is the ferrocene/ferrocenium redox couple, the potential of which has been measured in BMIOTf, and this is set as the zero reference potential, see Supporting Information). A clear peak is present in each histogram and can be used to calculate the conductance of the molecule. Figure 8 shows a two-dimensional (2-D) histogram representation of the data, with conductance on the ordinate, distance on the abscissa (corrected for the initial tip-substrate distance at the start of the $I(s)$ scan $),{ }^{58,60-62}$ and the color representing the point density ranging from dark blue (very low), through green to red (high point count). High point counts are seen at conductance values corresponding to the peak in the histogram, and also a high count tail is seen at the base of the plot (low conductance values). The latter corresponds to the break-off region; as the molecular bridge is stretched, the junction conductance drops, and the junction "snaps".

All of the conductance values obtained for 6pTTF6 over the range of electrochemical potentials studied are collected in Figure 9, which shows the molecular conductance vs the electrochemical sample potential (vs $\mathrm{Fc} / \mathrm{Fc}^{+}$). Conductance maxima of $\sim 2 \mathrm{nS}$ are observed for both redox transitions, while
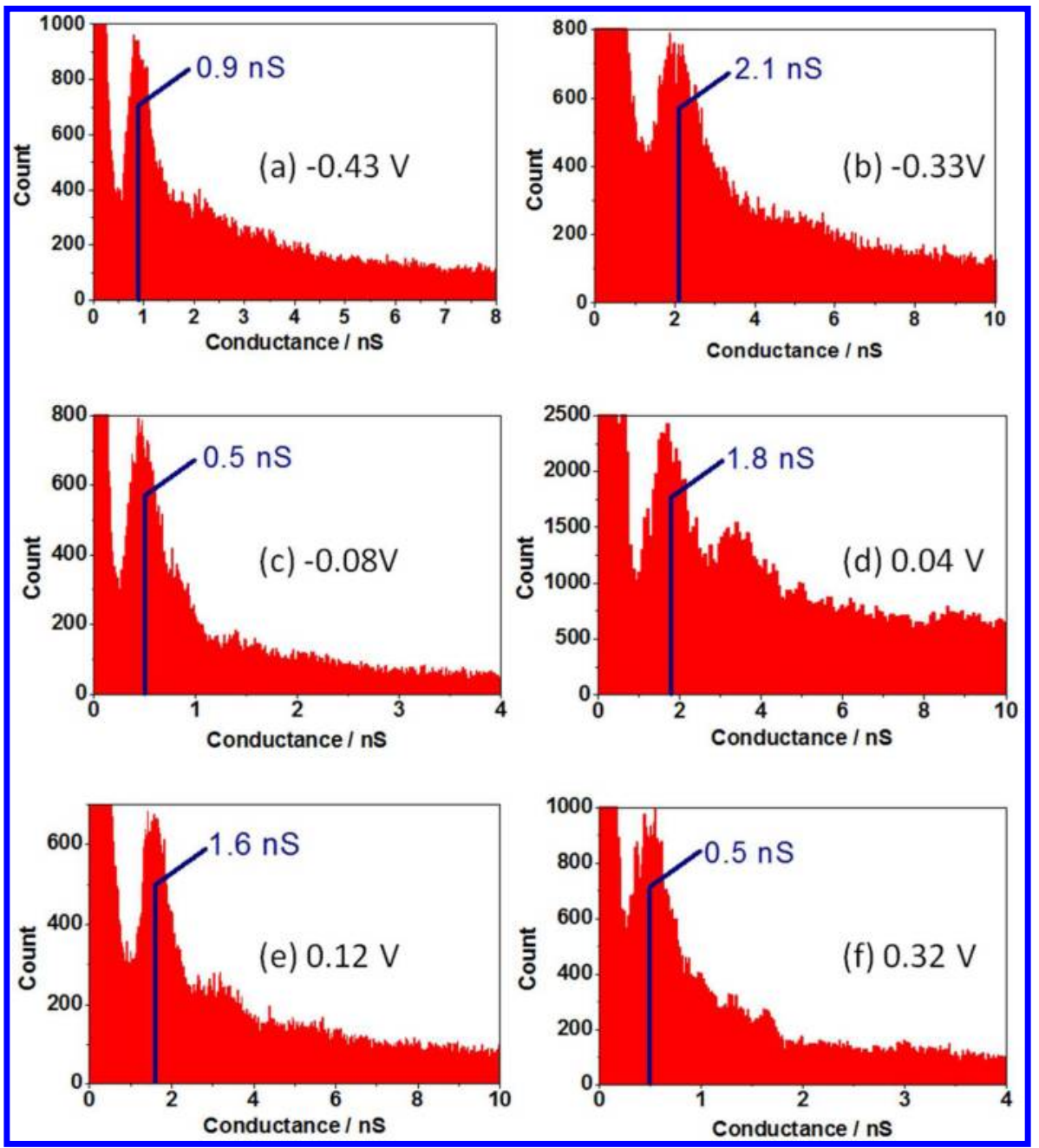

Figure 7. Conductance histograms of 6 TTTF6 using sample potentials of (a) $-0.43 \mathrm{~V}$, (b) $-0.33 \mathrm{~V}$, (c) $-0.08 \mathrm{~V}$, (d) $0.04 \mathrm{~V}$, (e) $0.12 \mathrm{~V}$, and (f) 0.32 $\mathrm{V}$ obtained using the $I(s)$ method; $V_{\text {bias }}=+0.6 \mathrm{~V} ; I_{0}=20 \mathrm{nA} ; 501,503,500,501,510$, and 503 scans were analyzed, respectively. Sample potentials are with respect to the $\mathrm{Fc} / \mathrm{Fc}^{+}$reference scale. 
(a) $-0.43 \mathrm{~V}$

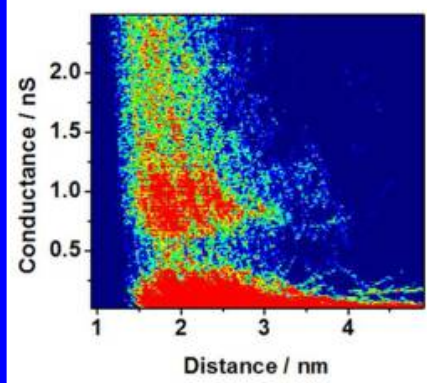

(c) $-0.08 \mathrm{~V}$

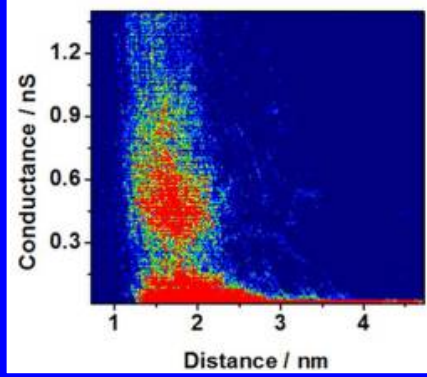

(b) $-0.33 \mathrm{~V}$

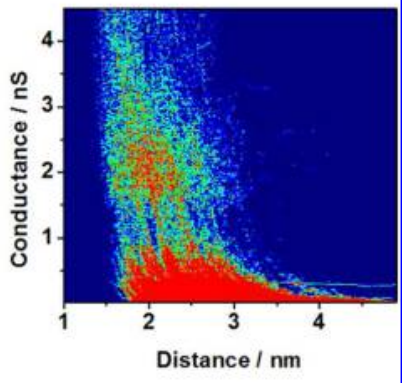

(d) $+0.12 \mathrm{~V}$

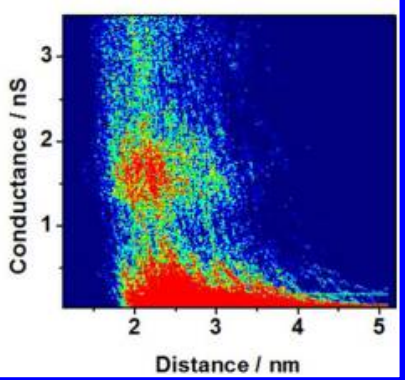

Figure 8. Two-dimensional (2-D) histogram representations of 6pTTF6 conductance data at sample potentials of (a) $-0.43 \mathrm{~V}$, (b) $-0.33 \mathrm{~V},(\mathrm{c})-0.08 \mathrm{~V}$, and $(\mathrm{d})+0.12 \mathrm{~V}$ vs the $\mathrm{Fc} / \mathrm{Fc}^{+}$reference scale. Data obtained using the $I(s)$ method.

the molecular conductance away from these redox transitions has the significantly smaller value of $\sim 0.5 \mathrm{nS}$.

The coincidence of the peaks in the conductance-electrode potential relation with the peaks in the voltammetric response point to electron transfer following a sequential two-step process with full or partial vibrational relaxation (KU model), rather than resonant tunneling. The latter would be expected to give a substantial noncoincidence of the peaks, with the offset along the potential axis expected to correspond to a reorganization free energy of generally at least several hundred millivolts (vide infra). To further explore the mechanism of charge transfer across the molecular bridge, the conductance data shown above was modeled using the sequential two-step (KU) model. Inherent in this model is a peak in the current flowing through the molecular bridge. ${ }^{29,63-65}$ In the case of 6pTTF6, the first step of the charge transfer occurs when the HOMO is close to the Fermi level of the "left hand" gold electrode, and involves hole tunneling to the HOMO of the pTTF group of the molecular bridge (or electron tunneling from the HOMO). The oxidized and vibrationally excited 6pTTF6 group then relaxes toward the Fermi level of the "right hand" gold electrode. However, if the electronic coupling between the molecule and the enclosing electrodes is strong enough and the adiabatic limit of interfacial electron/hole transfer prevails, the second charge transfer occurs before this relaxation is complete, and the hole tunnels to the right-hand electrode. The now fully occupied orbital then relaxes back toward to its original energy, where it can receive another hole, and the process repeats itself. Both a full version and a simplified numerical version of the KU model have been applied in the literature. The numerical form of the former is shown in eq 1 (see reference 29 and references cited therein and reference 13 for this numerical form of the KU equation):

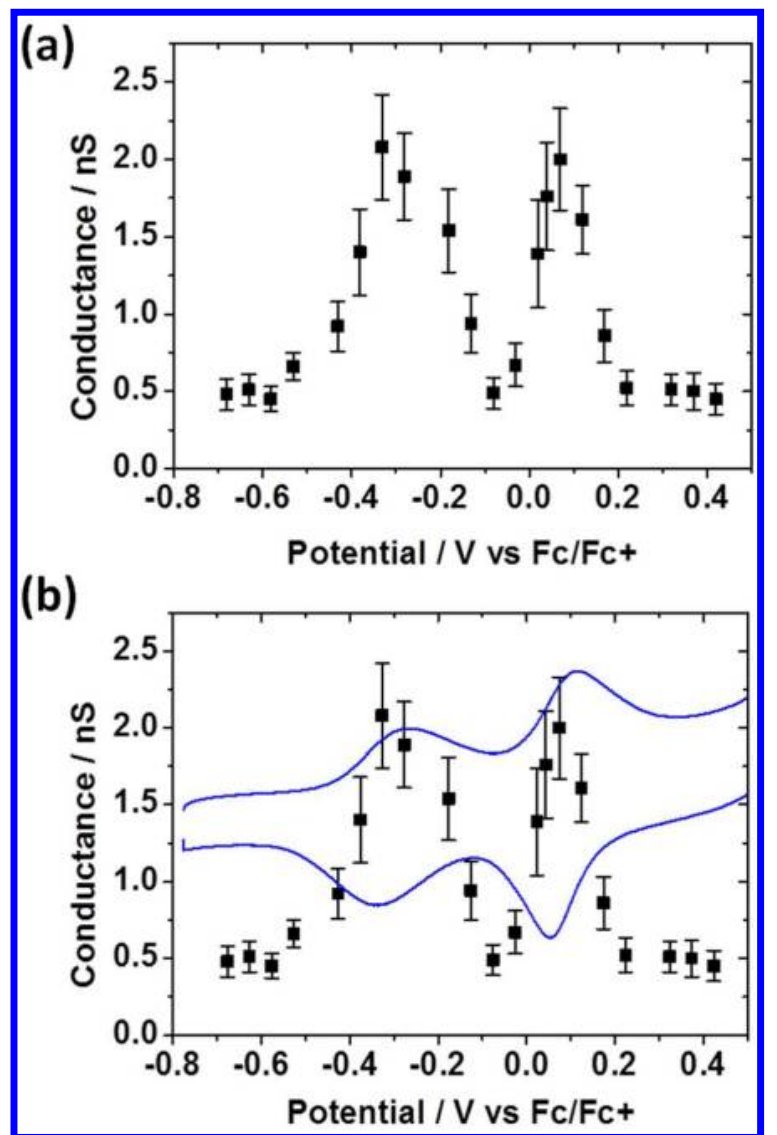

Figure 9. (a) Plot of conductance of 6pTTF6 against the sample electrochemical potential (with $V_{\text {bias }}=+0.6 \mathrm{~V}$ ) and (b) the plot in (a) overlaid with a cyclic voltammogram (blue line) of a 6pTTF6 monolayer. The point of maximum conductance corresponds with the redox potential of each redox transition of 6pTTF6.

$I_{\mathrm{e}}=1820 \cdot V_{\mathrm{BIAS}}\left\{\begin{array}{c}\exp \left[\frac{9.73}{\lambda_{\text {reorg }}}\left(\lambda_{\text {reorg }}+\xi e \eta+\gamma e V_{\mathrm{BIAS}}\right)^{2}\right]+ \\ \exp \left[\frac{9.73}{\lambda_{\text {reorg }}}\left(\lambda_{\text {reorg }}+e V_{\mathrm{BIAS}}-\xi e \eta-\gamma e V_{\mathrm{BIAS}}\right)^{2}\right]\end{array}\right\}$

where $I_{\mathrm{e}}$ is the enhanced current, $V_{\text {BIAS }}$ is the bias voltage, $\lambda_{\text {reorg }}$ is the total reorganization free energy, $\eta$ is the overpotential applied to the substrate, and $\xi$ and $\gamma$ are modeling parameters relating to the proportion of electrochemical potential and the bias potential respectively, that affect the redox moiety. All energy terms in the round brackets in eq 1 are expressed in units of electron volts; i.e. the term $e V_{\text {BIAS }}$ is numerically equivalent to the bias voltage. This equation can be simplified further to give: ${ }^{13}$

$$
I_{\mathrm{e}}=\frac{910 \cdot V_{\mathrm{BIAS}} \exp \left[-9.73\left(\lambda_{\text {reorg }}+e V_{\mathrm{BIAS}}\right)\right]}{\cosh \left[19.4\left(\xi e \eta+(\gamma-0.5) e V_{\mathrm{BIAS}}\right)\right]}
$$

The simplified form in eq 2 uses the assumption that $\eta$ and $V_{\text {BIAS }}$ are lower than $\lambda_{\text {reorg }}$ and it is simply derived from eq 1 using these assumptions. Both forms are used to model the ET properties of 6 pTTF6 and shall be referred to from this point as the "long KU" and "simplified KU" models, respectively. The best fits achieved using both the long and simplified KU for both redox transitions are shown in Table 1. The data fitting to both the long and simplified KU model are shown in Figure 10. 
Table 1. Values of $\lambda_{\text {reorg }}, \gamma$, and $\xi$ Used in the Modeling of Both Redox Transitions of 6pTTF6 Using the Long KU and Simplified KU Model of ET

\begin{tabular}{lccccc} 
& \multicolumn{2}{c}{ long KU } & & \multicolumn{2}{c}{ simplified KU } \\
\cline { 2 - 3 } \cline { 5 - 6 } \cline { 5 - 6 }$\lambda_{\text {reorg }} / \mathrm{eV}$ & first redox & second redox & & first redox & second redox \\
$\gamma$ & 1.165 & 1.18 & & 1.245 & 1.255 \\
$\xi$ & 0.5 & 0.5 & & 0.5 & 0.5 \\
& 0.8 & 1 & & 0.7 & 1
\end{tabular}

For both redox transitions of 6pTTF6, the fittings of both versions of the $\mathrm{KU}$ model match the conductance data well.

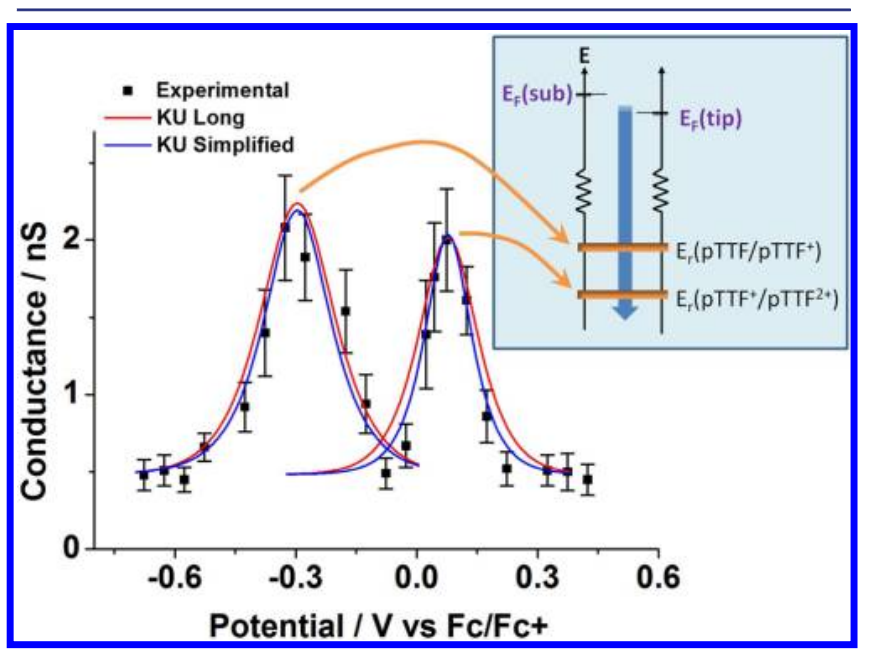

Figure 10. Conductance-sample potential relationship of 6pTTF6 in the RTIL BMIOTf. The red lines show the long KU model (eq 1), and the blue lines the simplified KU model (eq 2). For both redox transitions, both $\mathrm{KU}$ model versions fit the experimental data well. (Inset) Schematic energy level representation of the KU model for the two redox transitions. Varying the electrode potential (represented by the bold blue arrow) changes the relative energetic positions of the tip/substrate Fermi levels and the redox levels of pTTF. However, the bias voltage between gold tip and substrate remains constant as the electrode potential is changed. As the electrode potential is scanned positive, the $\mathrm{pTTF} / \mathrm{pTTF}^{+}$redox transition is first brought into "resonance", and then at more positive potentials the second redox transition $\left(\mathrm{pTTF}^{2+} / \mathrm{pTTF}^{+}\right)$comes into resonance.

$\lambda_{\text {reorg, }} \gamma$, and $\xi$ are used as variables in the fitting to the KU model. $\gamma$ is the shift of the effective electrode potential at the redox center with the variation of bias voltage. This should lie between 0 and 1 . Considering at first $\gamma$ and $\xi$ as free parameters, a good fitting is achieved for $\gamma=0.5$. This might seem reasonable, considering that the redox group is symmetrically placed in the junction for 6pTTF6, but see further below. Changing the $\gamma$ value shifts the peak of enhanced current along the $x$-axis, with a $\gamma$ smaller than 0.5 , resulting in the peak maximum at a negative overpotential $(\eta)$, and a $\gamma$ value larger than 0.5 , resulting in the peak maximum moving in a positive direction. The proportion of the electrochemical potential experienced at the redox site, $\xi$, can also have values ranging between 0 and 1 . The "ideal" $\xi$ value of 1 corresponds to the redox group fully experiencing changes in electrode potential. This may not be the case due to the double layer structuring and properties of the large ionic liquid ions in the confined nanoelectrode gap between the gold STM tip and substrate surface. Changing $\xi$ alters the width of the peak, with a higher $\xi$ approaching 1 giving a narrower peak and lower $\xi$ values resulting in a wider peak. In this instance, both types of $\mathrm{KU}$ model fit the experimental data for the second redox transition best, with the optimum $\xi$ value of 1 . For the first redox transition the best fit used $\xi$ values of 0.8 and 0.7 , for the long and simplified KU models, respectively.

The parameters $\gamma$ and $\xi$ are, however, correlated in the narrow tunneling gap in which the Debye length, $L_{\mathrm{d}}$ is comparable to the tunneling gap width $L$. The precise form of this correlation is not presently known for ionic liquids, but as an illustration, the correlation takes the following form for ionic solutions (say, aqueous solution) in the Debye-Hückel limit ${ }^{29,32,66}$

$$
\xi\left(z ; L_{\mathrm{d}}\right)=1-\gamma\left(L-z ; L_{\mathrm{d}}\right)-\gamma\left(z ; L_{\mathrm{d}}\right)
$$

where $z(\approx 0.5 L)$ is the position of the redox group counted from the working electrode surface. The Debye-Hückel limit has been shown to remain a good approximation also when finite size and lattice effects are taken into account, ${ }^{67-69}$ although these investigations only applied to the potential distribution in the tunneling gap when no redox group is present. In eq $4, \gamma\left(z ; L_{\mathrm{d}}\right)$ within the Debye-Hückel approximation takes the form

$$
\gamma\left(z ; L_{\mathrm{d}}\right)=\frac{\exp \left(\frac{z}{L_{\mathrm{d}}}\right)-\exp \left(-\frac{z}{L_{\mathrm{d}}}\right)}{\exp \left(\frac{L}{L_{\mathrm{d}}}\right)-\exp \left(-\frac{L}{L_{\mathrm{d}}}\right)}
$$

The width of the molecular conductance peak (in the conductance vs overpotential relationship), $\Delta$, is in the same approximation

$$
\frac{\Delta_{\eta}}{k_{\mathrm{B}} T}=\frac{4 \operatorname{arch}(2)}{1-\gamma\left(L-z ; L_{\mathrm{d}}\right)-\gamma\left(z ; L_{d}\right)}
$$

where $k_{\mathrm{B}}$ is Boltzmann's constant and $T$ the temperature. In the absence of a precise theoretical frame for the potential distribution in the tunneling gap for ionic liquids we can instead compare with eqs 3-5 in the limit of high solution ionic strength. This limit gives, for $z=0.5 L$ and $L_{\mathrm{d}} \ll L$

$$
\begin{aligned}
\gamma^{\text {high.ion }}\left(\frac{1}{2} L ; L_{\mathrm{d}}\right. & \ll L) \\
& \approx \exp \left(-\frac{1}{2} \frac{L}{L_{\mathrm{d}}}\right) \ll 1 \text { and } \\
& \frac{\Delta_{\eta}^{\text {high.ion }}}{k_{\mathrm{B}} T} \\
& \approx 4 \operatorname{arch}(2)
\end{aligned}
$$

$\xi \approx 1$ in this limit, eq 3 . We note that $\gamma=0.5$ is obtained at low ionic strength, $L_{\mathrm{d}} \geq L$ giving from eq $3 \xi \approx 0$. This is similar to the potential distribution at semi-infinite electrochemical surfaces.

Comparison between the free parametric fit of $\gamma$ and $\xi$ with a fit corresponding to the frame for correlated $\gamma$ and $\xi$ at high ionic strength (say for $\gamma \approx 0.1$ and $\xi \approx 1$ ) shows however that, although the shape of the conductance peak is maintained, the peak maximum is significantly shifted toward negative overpotentials (by about a couple of hundred millivolts). Figure 8 shows that small negative shifts of the conductance peak compared with the voltammetric peaks perhaps cannot be excluded, but these are much smaller than what emerges from 
the simulation when $\gamma$ is changed from a value of about 0.5 to a value of about 0.1 . Reasons for this apparent discrepancy are undoubtedly associated with the large physical size of the ionic liquid ions, the size of which compares with that of both the redox center and the tunneling gap width. Precise assessment of the potential distribution in the tunneling gap is therefore not immediately represented by continuum models even when finite size effects such as those addressed in references 68 and 69 are included.

These observations may also have a bearing on the observed conductance vs overpotential peak widths. The peak widths are around $200 \mathrm{mV}$ which is larger than the value of $5.2 \times k_{\mathrm{B}} T \approx$ $130 \mathrm{mV}$ expected in the limit of high solute ionic strength, eq 6 . Although apparently conspicuous, this difference is not, however, significant enough to disclose a discrepancy from the sequential two-step view of the two 6pTTF6 oxidative transitions. For one thing the potential distribution in the tunneling gap and the overpotential dependence of this distribution remains somewhat elusive considering the strong finite-size effects of the ionic liquid ions as noted. In addition, the large bias voltage $(0.6 \mathrm{~V})$ needed to enhance the singlemolecule conductance values is also at the brink where bias voltage convolution of the conductance peak width may begin. The physical origin of this broadening effect is rooted in the increasing contribution of electronic energy levels below the Fermi levels of the enclosing working electrode and in situ STM tip as the bias voltage is increased.

Changing the reorganization free energy $\lambda_{\text {reorg }}$ changes the height of the peak for the enhanced current; a lower $\lambda_{\text {reorg }}$ results in a larger enhanced current and vice versa. Using the long KU model, $\lambda_{\text {reorg }}$ was estimated to be 1.17 and $1.18 \mathrm{eV}$ for the first and second redox transitions, respectively. Using the simplified KU model, $\lambda_{\text {reorg }}$ values of 1.25 and $1.26 \mathrm{eV}$ were estimated for the first and second redox transitions. From this it can be noted that $\lambda_{\text {reorg }}$ for both redox transitions are very similar using each KU model. $\lambda_{\text {reorg }}$ consists of two parts, $\lambda_{\text {intra }}$ and $\lambda_{\text {solv }}$ which describe the inner-sphere and outer-sphere interactions, respectively. $\lambda_{\text {intra }}$ of 6pTTF6 has not been calculated. However, various other TTF derivatives have been investigated and found to have a $\lambda_{\text {intra }}$ between 200 and 600 meV. ${ }^{70-73}$ It is a reasonable assumption that $\lambda_{\text {intra }}$ of 6 pTTF6 would be similar to other TTFs. When the solvent is a RTIL, $\lambda_{\text {solv }}$ is expected to be large. ${ }^{74-76}$ Accurately computing $\lambda_{\text {solv }}$ for the molecular bridge solvated in the ionic liquid and within the nanogap environment would be a very demanding challenge, which is beyond the scope of this report, but $\lambda_{\text {solv }}$ can be estimated (crudely) on the basis of dielectric continuum views. For interfacial electrochemical electron transfer in a semiinfinite dielectric medium

$$
\lambda_{\text {solv }}=\left(\frac{e^{2}}{8 \pi \varepsilon_{0}}\right)\left(\frac{1}{a}-\frac{1}{2 r}\right)\left(\frac{1}{n^{2}}-\frac{1}{\varepsilon_{\mathrm{r}}}\right)
$$

where $\varepsilon_{0}$ is the permittivity of free space, $a$ the ionic radius, and $r$ the distance between the center of the redox group and the electrode surface (which is taken to be $1.43 \mathrm{~nm}$ ), $n$ is the refractive index of the solvent $(1.434),{ }^{77}$ and $\varepsilon_{\mathrm{r}}$ is the relative permittivity of the solvent (12.9), ${ }^{78}$ which in this instance is BMIOTf. ${ }^{79,80}$ For the purpose of our approximation, in eq $7 a$ is set at half the distance between the nitrogen atoms in the redox active moiety of the 6pTTF6 molecular bridge. This produces a $\lambda_{\text {solv }}$ value of $\sim 0.46 \mathrm{eV}$. This very approximate value of $\lambda_{\text {solv }}$ when added to an assumed value between 0.2 and $0.6 \mathrm{eV}$ for $\lambda_{\text {intra }}$ results in a $\lambda_{\text {reorg }}$ value similar to that observed for 6pTTF6 in a BMIOTf solvent using the KU model.

The amount of solvent reorganized in the tunneling gap is, however, significantly smaller than in the semi-infinite dielectric medium of electrochemical processes. This confinement effect has been analyzed in some detail using a dielectric continuum view combined with a metallic spherical model for the redox group and a metallic hemispherical model for the STM tip. ${ }^{81}$ This analysis showed that $\lambda_{\text {solv }}$ is reduced by $15-30 \%$, depending on the gap width and the radii of the molecule and tip, compared with the semi-infinite dielectric medium. This would correspond to a reduction of $\lambda_{\text {solv }}$ from about 0.46 $\mathrm{eV}$ to between about $0.39-0.32 \mathrm{eV}$ for the present system.

To shed further light on the experimentally determined value of reorganization free energy for pTTF in ionic liquid, we have contrasted it with $\lambda_{\text {reorg }}$ obtained for pTTF from singlemolecule conductance data in aqueous electrolytes. Both the long and simplified KU models have been used to model the ET through 6pTTF6 during the first redox transition in an aqueous buffer electrolyte. The resulting fit values for $\lambda_{\text {reorg }} \gamma$, and $\xi$ are shown in Figure 11, and the parameters used in the

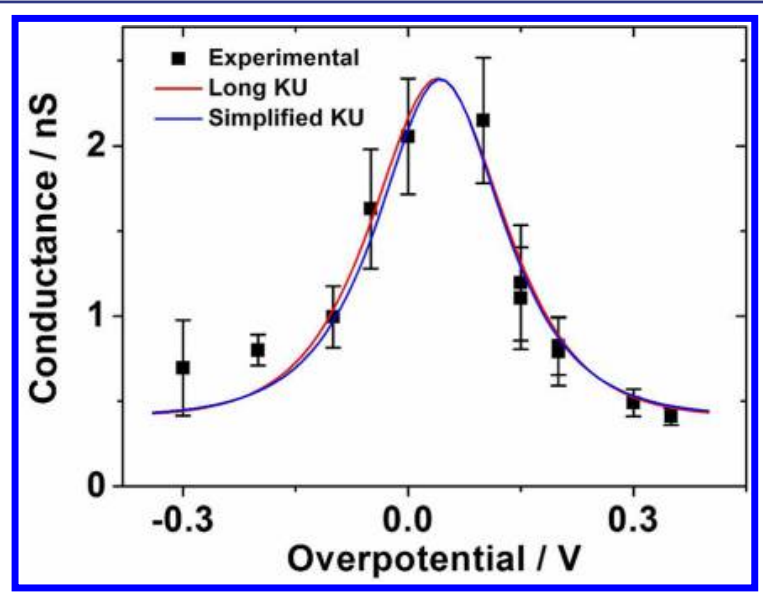

Figure 11. Conductance-overpotential relationship of 6pTTF6 in an aqueous buffer electrolyte at $\mathrm{pH} 6.8$, recorded by Leary et al. ${ }^{22}$ with $V_{\text {bias }}=0.2 \mathrm{~V}$. The red line shows the long KU model (eq 1), and the blue line the simplified KU model (eq 2). $\lambda_{\text {reorg }}$ of 6pTTF6 in an aqueous buffer was estimated as $0.41 \mathrm{eV}$ using the long $\mathrm{KU}$ model and $0.43 \mathrm{eV}$ using the simplified KU model.

KU modeling are summarized in Table 2. The values of $\lambda_{\text {reorg }}$ are much lower for 6pTTF6 in the aqueous electrolyte than in BMIOTf. This is expected, as the aqueous buffer electrolyte expends much less energy reorganizing around the 6pTTF6 molecule as the redox state changes.

Table 2. Values of $\lambda_{\text {reorg, }} \gamma$, and $\xi$ Used in the Modeling of 6pTTF6 Single-Molecule Conductance Recorded in Aqueous Electrolyte by Leary et al. ${ }^{22 a}$

\begin{tabular}{lcc} 
& \multicolumn{2}{c}{ 6pTTF6 in aqueous electrolyte } \\
\cline { 2 - 3 } & long KU & short KU \\
$\lambda_{\text {reorg }} / \mathrm{eV}$ & 0.41 & 0.43 \\
$\gamma$ & 0.4 & 0.35 \\
$\xi$ & 0.5 & 0.7
\end{tabular}

${ }^{a_{T}}$ The simulated values listed are for the "long" and "simplified" numerical versions of the KU model (see text). 


\section{CONCLUSIONS}

The single-molecule conductance of the redox active molecular wire 6pTTF6 has been recorded using electrochemical potential control in the RTIL BMIOTf. Ranges of electrochemical potentials were investigated, and the conductance of 6pTTF6 was followed over the two redox transitions it undergoes. Both conventional and 2-D conductance histograms have been constructed. Taking advantage of the wider potential window available to BMIOTf over conventional aqueous electrolytes, both redox transitions of 6pTTF6 were accessible in both cyclic voltammetry and notably in STM $I(s)$ measurements. Two separated transitions in the same molecule have not been reported before by the in situ $I(s)$ or BJ measurements (although single peaks for multiple singleelectron charging events have been reported ${ }^{16}$ ). In this respect ionic liquids are offering new perspectives for studying electrochemical conductance gating across multiple redox states of the target redox molecule. By contrast, in aqueous electrolytes, only the first redox transition, from $6 \mathrm{pTTF}^{0}$ to 6 pTF6 $^{+\bullet}$ is observed. $^{22}$

An increase in the single-molecule conductance of 6pTTF6 from $\sim 0.5$ to $\sim 2 \mathrm{nS}$ was observed, around the redox potential for both redox transitions in the ionic liquid. The conductance data obtained for 6pTTF6 in BMIOTf could be modeled by the KU model of two-step charge transfer with partial vibrational relaxation, which provided a good fit to the data. $\lambda_{\text {reorg }}$ was estimated to be $\sim 1.2 \mathrm{eV}$ for both redox transitions in BMIOTf, compared to $\sim 0.4 \mathrm{eV}$ for the first redox transition in an aqueous electrolyte, recorded by Leary et al. ${ }^{22}$ This is attributed to the large contribution of $\lambda_{\text {solv }}$ in the RTIL. This work shows some of the advantages of using a RTIL as a medium for singlemolecule conductance measurements, particularly the wider electrochemical potential window. It is expected that RTILs will be more widely deployed in the future in the field of singlemolecule electronics since they open avenues for studying redox transitions not readily accessible in aqueous electrolytes.

\section{ASSOCIATED CONTENT}

\section{S Supporting Information}

Additional voltammetric data and surface infrared spectroscopy (PM-IRRAS) of 6pTTF6. This material is available free of charge via the Internet at http://pubs.acs.org.

\section{AUTHOR INFORMATION}

\section{Corresponding Author}

nichols@liv.ac.uk (R.J.N.); shiggins@liv.ac.uk (S.J.H.).

\section{Present Address}

${ }^{\perp}$ Departamento de Física de la Materia Condensada and Instituto "Nicolas Cabrera", Universidad Autonoma de Madrid, E-28049 Madrid, Spain, and Instituto Madrileño de Estudios Advanzados en Nanociencia (IMDEA-Nanociencia), Ciudad Universitaria de Cantoblanco, E-28049 Madrid, Spain.

\section{Notes}

The authors declare no competing financial interest.

\section{ACKNOWLEDGMENTS}

J.O.J. gratefully acknowledges financial support provided by the Villum Foundation and the Danish Natural Science Research Council (\#11-106744). The Liverpool group acknowledges EPSRC for financial support and the Otto Mønsted Foundation for an Otto Mønsted Visiting Professorship to R.J.N.

\section{REFERENCES}

(1) Kubatkin, S.; Danilov, A.; Hjort, M.; Cornil, J.; Bredas, J. L.; Stuhr-Hansen, N.; Hedegard, P.; Bjornholm, T. Nature 2003, 425, 698.

(2) Osorio, E. A.; Bjornholm, T.; Lehn, J. M.; Ruben, M.; van der Zant, H. S. J. I. Phys.: Condens. Matter 2008, 20, 374121.

(3) van der Zant, H. S. J.; Kervennic, Y. V.; Poot, M.; O’Neill, K.; de Groot, Z.; Thijssen, J. M.; Heersche, H. B.; Stuhr-Hansen, N.; Bjornholm, T.; Vanmaekelbergh, D.; van Walree, C. A.; Jenneskens, L. W. Faraday Discuss. 2006, 131, 347.

(4) Osorio, E. A.; O’Neill, K.; Stuhr-Hansen, N.; Nielsen, O. F.; Bjornholm, T.; van der Zant, H. S. I. Adv. Mater. 2007, 19, 281.

(5) Osorio, E. A.; O’Neill, K.; Wegewijs, M.; Stuhr-Hansen, N.; Paaske, J.; Bjornholm, T.; van der Zant, H. S. J. Nano Lett. 2007, 7, 3336.

(6) Champagne, A. R.; Pasupathy, A. N.; Ralph, D. C. Nano Lett. 2005, 5, 305.

(7) Di Ventra, M.; Lang, N. D.; Pantelides, S. T. Chem. Phys. 2002, $281,189$.

(8) Tao, N. I. Phvs. Rev. Lett. 1996, 76, 4066.

(9) Xu, B. Q.; Tao, N. J. I. Science 2003, 301, 1221.

(10) Haiss, W.; van Zalinge, H.; Higgins, S. J.; Bethell, D.; Hobenreich, H.; Schiffrin, D. J.; Nichols, R. J. J. Am. Chem. Soc. 2003, 125, 15294.

(11) Haiss, W.; van Zalinge, H.; Hobenreich, H.; Bethell, D.; Schiffrin, D. J.; Higgins, S. J.; Nichols, R. I. Langmuir 2004, 20, 7694.

(12) Haiss, W.; Albrecht, T.; van Zalinge, H.; Higgins, S. J.; Bethell, D.; Hoebenreich, H.; Schiffrin, D. J.; Nichols, R. J.; Kuznetsov, A. M.; Zhang, J.; Chi, Q.; Ulstrup, J. I. Phvs. Chem. B 2007, 111, 6703.

(13) Pobelov, I. V.; Li, Z.; Wandlowski, T. J. Am. Chem. Soc. 2008, 130, 16045 .

(14) Li, Z.; Pobelov, I.; Han, B.; Wandlowski, T.; Blaszczyk, A.; Mayor, M. Nanotechnology 2007, 18, 044018.

(15) Li, Z.; Han, B.; Meszaros, G.; Pobelov, I.; Wandlowski, T.; Blaszczyk, A.; Mayor, M. Faradav Discuss. 2006, 131, 121.

(16) Li, X. L.; Xu, B. Q.; Xiao, X. Y.; Yang, X. M.; Zang, L.; Tao, N. J. Faradav Discuss. 2006, 131, 111

(17) Li, X.; Hihath, J.; Chen, F.; Masuda, T.; Zang, L.; Tao, N. J. Am. Chem. Soc. 2007, 129, 11535.

(18) Li, C.; Mishchenko, A.; Li, Z.; Pobelov, I.; Wandlowski, T.; Li, X. Q.; Wuerthner, F.; Bagrets, A.; Evers, F. J. Phys.: Condens. Matter 2008, 20, 374122.

(19) Chen, F.; He, J.; Nuckolls, C.; Roberts, T.; Klare, J. E.; Lindsay, S. Nano Lett. 2005, 5, 503.

(20) Chen, F.; Nuckolls, C.; Lindsay, S. Chem. Phvs. 2006, 324, 236.

(21) He, J.; Chen, F.; Lindsay, S.; Nuckolls, C. Appl. Phys. Lett. 2007, $90,072112$.

(22) Leary, E.; Higgins, S. J.; van Zalinge, H.; Haiss, W.; Nichols, R. J.; Nygaard, S.; Jeppesen, J. O.; Ulstrup, J. I. Am. Chem. Soc. 2008, 130, 12204.

(23) Albrecht, T.; Moth-Poulsen, K.; Christensen, J. B.; Hjelm, J.; Bjornholm, T.; Ulstrup, J. I. Am. Chem. Soc. 2006, 128, 6574.

(24) Albrecht, T.; Moth-Poulsen, K.; Christensen, J. B.; Guckian, A.; Bjornholm, T.; Vos, J. G.; Ulstrup, J. Faradav Discuss. 2006, 131, 265.

(25) Albrecht, T.; Guckian, A.; Kuznetsov, A. M.; Vos, J. G.; Ulstrup, I. I. Am. Chem. Soc. 2006, 128, 17132.

(26) Albrecht, T.; Guckian, A.; Ulstrup, J.; Vos, J. G. IEEE Trans. Nanotechnol. 2005, 4, 430.

(27) Albrecht, T.; Guckian, A.; Ulstrup, J.; Vos, J. G. Nano Lett. 2005, $5,1451$.

(28) Chi, Q. J.; Farver, O.; Ulstrup, J. Proc. Natl. Acad. Sci. U.S.A 2005, 102, 16203.

(29) Zhang, J.; Kuznetsov, A. M.; Medvedev, I. G.; Chi, Q.; Albrecht, T.; Jensen, P. S.; Ulstrup, J. Chem. Rev. 2008, 108, 2737.

(30) Shu, C.; Li, C. Z.; He, H. X.; Bogozi, A.; Bunch, J. S.; Tao, N. J. Phvs. Rev. Lett. 2000, 84, 5196.

(31) Zhang, J.; Chi, Q.; Kuznetsov, A. M.; Hansen, A. G.; Wackerbarth, H.; Christensen, H. E. M.; Andersen, J. E. T.; Ulstrup, J. J. Phys. Chem. B 2002, 106, 1131. 
(32) Kuznetsov, A. M.; Medvedev, I. G.; Ulstrup, J. J. Chem. Phys. 2007, 127

(33) Kuznetsov, A. M.; Ulstrup, J. Surf. Coat. Technol. 1994, 67, 193.

(34) Kuznetsov, A. M.; Ulstrup, J. L. Phvs. Chem. A 2000, 104, 11531.

(35) Zhang, J. D.; Kuznetsov, A. M.; Ulstrup, J. J. Electroanal. Chem. 2003, 541, 133.

(36) Zhang, J. D.; Chi, Q. J.; Albrecht, T.; Kuznetsov, A. M.; Grubb, M.; Hansen, A. G.; Wackerbarth, H.; Welinder, A. C.; Ulstrup, J. Electrochim. Acta 2005, 50, 3143.

(37) Schmickler, W.; Tao, N. I. Electrochim. Acta 1997, 42, 2809.

(38) Schmickler, W.; Widrig, C. L. Electroanal. Chem. 1992, 336, 213.

(39) O’Boyle, N. M.; Albrecht, T.; Murgida, D. H.; Cassidy, L.; Ulstrup, J.; Vos, J. G. Inorg. Chem. 2007, 46, 117.

(40) Becher, J.; Jeppesen, J. O.; Nielsen, K. Synth. Met. 2003, 133, 309.

(41) Jeppesen, J. O.; Becher, J. Eur. I. Org. Chem. 2003, 3245.

(42) Giacalone, F.; Herranz, M. A.; Grueter, L.; Gonzalez, M. T.; Calame, M.; Schoenenberger, C.; Arroyo, C. R.; Rubio-Bollinger, G.; Velez, M.; Agrait, N.; Martin, N. Chem. Commun. 2007, 4854.

(43) Zhang, S.; Leem, G.; Lee, T. R. Langmuir 2009, 25, 13855.

(44) Kobayashi, Y.; Yoshioka, M.; Saigo, K.; Hashizume, D.; Ogura, T. I. Am. Chem. Soc. 2009, 131, 9995.

(45) Olaya, A. J.; Ge, P.; Gonthier, J. F.; Pechy, P.; Corminboeuf, C.; Girault, H. H. I. Am. Chem. Soc. 2011, 133, 12115.

(46) Aldous, L.; Silvester, D. S.; Villagran, C.; Pitner, W. R.; Compton, R. G.; Lagunas, M. C.; Hardacre, C. New J. Chem. 2006, 30, 1576.

(47) Fu, Y.-C.; Zhang, H.-M.; Su, Y.-Z.; Wu, D.-Y.; Xie, Z.-X.; Mao,

B.-W. Z. Phys. Chem. 2007, 221, 1109-1121.

(48) Ji, X.; Silvester, D. S.; Aldous, L.; Hardacre, C.; Compton, R. G. I. Phvs. Chem. C 2007, 111, 9562.

(49) Lin, L.-G.; Yan, J.-W.; Wang, Y.; Fu, Y.-C.; Mao, B.-W. J. Exp. Nanosci. 2006, 1, 269.

(50) Buzzeo, M. C.; Evans, R. G.; Compton, R. G. ChemPhysChem 2004, 5, 1106.

(51) Endres, F.; El Abedin, S. Z. Phys. Chem. Chem. Phys. 2006, 8, 2101

(52) Carstens, T.; El Abedin, S. Z.; Endres, F. ChemPhysChem 2008, 9, 439 .

(53) Fu, Y.-C.; Su, Y.-Z.; Zhang, H.-M.; Yan, J.-W.; Xie, Z.-X.; Mao, B.-W. Electrochim. Acta 2010, 55, 8105.

(54) Mukhopadhyay, I.; Aravinda, C. L.; Borissov, D.; Freyland, W. Electrochim. Acta 2005, 50, 1275.

(55) Freyland, W.; Zell, C. A.; El Abedin, S. Z.; Endres, F. Electrochim. Acta 2003, 48, 3053.

(56) Fu, Y.-C.; Yan, J.-W.; Wang, Y.; Tian, J.-H.; Zhang, H.-M.; Xie, Z.-X.; Mao, B.-W. I. Phvs. Chem. C 2007, 111, 10467.

(57) Lin, L. G.; Wang, Y.; Yan, J. W.; Yuan, Y. Z.; Xiang, J.; Mao, B. W. Electrochem. Commun. 2003, 5, 995.

(58) Kay, N. J.; Nichols, R. J.; Higgins, S. J.; Haiss, W.; Sedghi, G.; Schwarzacher, W.; Mao, B.-W. I. Phvs. Chem. C 2011, 115, 21402.

(59) Doi, I.; Miyazaki, E.; Takimiya, K. Chem. Lett. 2008, 37, 1088.

(60) Martin, S.; Haiss, W.; Higgins, S.; Cea, P.; Lopez, M. C.; Nichols, R. J. I. Phvs. Chem. C 2008, 112, 3941.

(61) Haiss, W.; Wang, C.; Grace, I.; Batsanov, A. S.; Schiffrin, D. J.; Higgins, S. J.; Bryce, M. R.; Lambert, C. J.; Nichols, R. J. Nat. Mater. 2006, 5, 995.

(62) Sedghi, G.; Sawada, K.; Esdaile, L. J.; Hoffmann, M.; Anderson, H. L.; Bethell, D.; Haiss, W.; Higgins, S. J.; Nichols, R. J. J. Am. Chem. Soc. 2008, 130, 8582 .

(63) Kuznetsov, A. M.; Ulstrup, J. J. Electroanal. Chem. 2004, 564, 209.

(64) Kuznetsov, A. M.; Ulstrup, J. I. Chem. Phvs. 2002, 116, 2149.

(65) Kuznetsov, A. M.; Ulstrup, J. Russ. J. Electrochem. 2006, 42, 760.

(66) Medvedev, I. G. Electrochim. Acta 2008, 53, 6545.

(67) Kornyshev, A. A.; Kuznetsov, A. M. ChemPhysChem 2006, 7, 1036.

(68) Kornyshev, A. A.; Kuznetsov, A. M. Chem. Phvs. 2006, 324, 276.
(69) Kornyshev, A. A.; Kuznetsov, A. M.; Ulstrup, J. Proc. Natl. Acad. Sci. U.S.A 2006, 103, 6799.

(70) Casado, J.; Zgierski, M. Z.; Ruiz Delgado, M. C.; Lopez Navarrete, J. T.; Mas-Torrent, M.; Rovira, C. J. Phys. Chem. C 2007, $111,10110$.

(71) Li, H.-x.; Zheng, R.-h.; Shi, Q. Phys. Chem. Chem. Phys. 2011, 13, 5642.

(72) Bromley, S. T.; Mas-Torrent, M.; Hadley, P.; Rovira, C. J. Am. Chem. Soc. 2004, 126, 6544.

(73) Mas-Torrent, M.; Hadley, P.; Bromley, S. T.; Ribas, X.; Tarres, J.; Mas, M.; Molins, E.; Veciana, J.; Rovira, C. J. Am. Chem. Soc. 2004, $126,8546$.

(74) Shim, Y.; Duan, J. S.; Choi, M. Y.; Kim, H. J. J. Chem. Phys. 2003, 119, 6411-6414.

(75) Shim, Y.; Choi, M. Y.; Kim, H. J. J. Chem. Phys. 2005, 122, 044510 .

(76) Maginn, E. J. I. Phvs.: Condens. Matter 2009, 21, 373101.

(77) Shamsipur, M.; Beigi, A. A. M.; Teymouri, M.; Pourmortazavi, S. M.; Irandoust, M. I. Mol. Lia. 2010, 157, 43.

(78) Huang, M.-M.; Jiang, Y.; Sasisanker, P.; Driver, G. W.; Weingartner, H. I. Chem. Eng. Data 2011, 56, 1494.

(79) Marcus, R. A. Angew. Chem. Int. Ed. 1993, 32, 1111.

(80) Liang, M.; Kaintz, A.; Baker, G. A.; Maroncelli, M. J. Phys. Chem. B 2012, 116, 1370.

(81) Friis, E. P.; Kharkats, Y. I.; Kuznetsov, A. M.; Ulstrup, J. J. Phys. Chem. A 1998, 102, 7851.

(82) Gnahm, M.; Kolb, D. M. L. Electroanal. Chem. 2011, 651, 250.

(83) Hultgren, V. M.; Mariotti, A. W. A.; Bond, A. M.; Wedd, A. G. Anal. Chem. 2002, 74, 3151. 